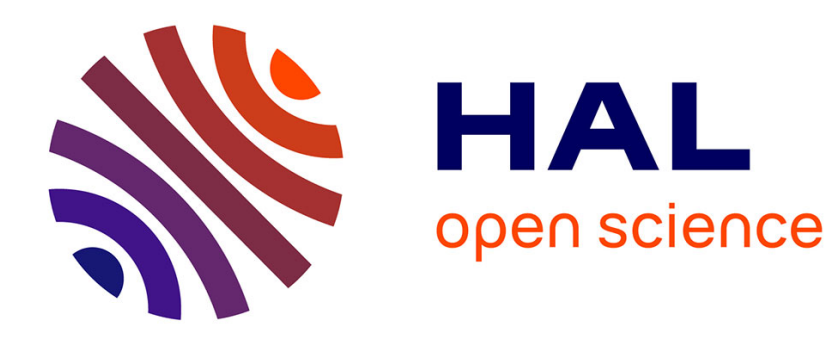

\title{
L'augmentation du patrimoine urbain, une exploration informationnelle
}

\author{
Jessica de Bideran
}

\section{To cite this version:}

Jessica de Bideran. L'augmentation du patrimoine urbain, une exploration informationnelle. Sciences du Design, 2016, 3, pp.57-68. hal-01855791

\section{HAL Id: hal-01855791 \\ https://hal.science/hal-01855791}

Submitted on 8 Aug 2018

HAL is a multi-disciplinary open access archive for the deposit and dissemination of scientific research documents, whether they are published or not. The documents may come from teaching and research institutions in France or abroad, or from public or private research centers.
L'archive ouverte pluridisciplinaire HAL, est destinée au dépôt et à la diffusion de documents scientifiques de niveau recherche, publiés ou non, émanant des établissements d'enseignement et de recherche français ou étrangers, des laboratoires publics ou privés. 


\title{
L'augmentation du patrimoine urbain, une exploration informationnelle
}

\author{
Jessica de Bideran
}

\begin{abstract}
RÉSUMÉ
Pour accompagner dans leur découverte patrimoniale les nombreux visiteurs qui sillonnent aujourd'hui la ville de Bordeaux, un Centre d'Interprétation de l'Architecture et du Patrimoine a récemment été ouvert sur la place de la Bourse. Durant l'été 2015, cette exposition permanente s'est étendue à l'extérieur par la mise à disposition d'une application de visite en réalité augmentée. Désirant transporter le public au début du $\mathrm{XX}^{\mathrm{e}}$ siècle, cette nouvelle forme de valorisation patrimoniale construit une expérience spatiale de la ville qui mêle positionnement topographique au cœur d'un patrimoine urbain avec des navigations au sein d'un univers numérique. L'analyse précise de cette application et du dispositif d'exposition associé permet de questionner le processus d'appropriation de l'espace public patrimonial à l'ère du numérique. En nous concentrant sur les interactions proposées nous constaterons combien, pour produire du sens, le design doit non seulement prendre en compte les pratiques du visiteur mais aussi l'ensemble de l'écosystème de la visite patrimoniale.
\end{abstract}

MOTS-CLÉS : Réalité augmentée, dispositif de visite, design d'interaction, patrimoine urbain, visite patrimoniale.

\begin{abstract}
In Bordeaux, on the famous place de la Bourse, a « Center of Interpretation of the Architecture and the Heritage » open recently in order to guide every curious who visit the city. During the 2015 summer, the permanent exhibition of the Center extend to virtual reality by an first ever application. Wishing to take visitors back at the beginning of the XXth century, this patrimonial writing develop a spatial experiment of the city which combine topographic positioning with urban heritage and navigations in a digital universe. By analysing the application and the associated plan of exhibition, we can questioning the process of appropriation of the public patrimonial area in our digital age. In light of the proposed interactions, we shall notice how much, to produce sense, the design has to consider visitor's practices but also has to think the whole ecosystem of patrimonial visits.
\end{abstract}

KEYWORDS : Augmented reality, device of visit, design of interaction, urban heritage, patrimonial visit 


\section{Introduction}

Classée par l'Unesco au patrimoine mondial de 1'Humanité en 2007, la ville de Bordeaux est depuis sillonnée de visiteurs. Pour les accompagner dans leur découverte urbaine, un Centre d'Interprétation de l'Architecture et du Patrimoine (Ciap) a récemment été ouvert sur la place de la Bourse, en plein cœur du secteur inscrit au patrimoine mondial. Inauguré en 2014, cet espace institutionnel présente la construction et l'évolution du tissu urbain de la cité bordelaise, de ces origines protohistoriques aux grands chantiers à venir du $\mathrm{XXI}^{\mathrm{e}}$ siècle, à l'aide notamment de nombreux dispositifs numériques de valorisation documentaire (films, bases de données et applications interactives). Durant l'été 2015, l'exposition s'est étendue à l'extérieur par la création et la mise à disposition pour ces publics d'une application de visite en réalité augmentée, selon l'expression des producteurs et commanditaires, dont l'objectif était de transporter son usager au début du $\mathrm{XX}^{\mathrm{e}}$ siècle. Intitulée «Bordeaux, passé augmenté », cette application a été produite par le studio de design interactif 2Rroqs grâce au soutien du Ministère de la Culture et de la Communication et de la ville de Bordeaux dans le cadre de l'appel à projets 2014 «Services numériques culturels innovants ».

Si depuis quelques années, les applications numériques de visite se multiplient, les publications scientifiques s'intéressant spécifiquement à ce domaine sont encore peu nombreuses alors même que la «ville numérique »semble un sujet fécond (Pagès, 2010). Des tendances ainsi que des formes de médiation ont par exemple pu être dressées à partir de l'étude de dispositifs se déployant à l'intérieur de lieux culturels (Lesaffre, Watremez et Flon, 2014). Plus rares sont les analyses portant sur des outils conçus à l'échelle de centres urbains (Bordeaux et Renaud, 2012 et 2014 ; Bideran, 2014). Nous souhaitons donc contribuer à ces réflexions en tentant de mieux saisir le processus d'appropriation de l'espace public patrimonial qu'élabore «Bordeaux, passé augmenté ».

Concrètement, ce dispositif propose à tout visiteur muni d'un smartphone ou d'une tablette d'accéder à des photographies anciennes représentant des lieux emblématiques de la ville de Bordeaux, photographies animées grâce un traitement multi-plan réalisé dans un logiciel développé ad hoc. Grâce à l'interface numérique, le visiteur franchit la surface plane de la carte postale ancienne et pénètre à l'intérieur d'un document mis en volume, animé et sonorisé, où il devient maître de ses déplacements. Le système d'exposition proposé construit ainsi une expérience de la ville qui hybride positionnement topographique au sein de la cité et interactions avec un dispositif numérique qui propose à l'usager une autre représentation de cet espace urbain. Or, en tant que système de réflexion et de production ayant engendré ce dispositif, le design construit du sens qu'il communique à l'usager via l'objet et les interactions proposées (Frechin, 2008). Décortiquer ce design amène par conséquent à interroger les représentations véhiculées par l'outil tout autant que la pratique de l'espace urbain qu'il propose au visiteur. Comment la navigation au sein de l'espace numérique représenté s'articule-t-elle avec celle, plus traditionnelle, qu'expérimente le visiteur au sein du lieu physique et monumental ? Dans quelle mesure l'expérience de la visite patrimoniale se voit-elle augmentée ?

Pour répondre à ces questions, nous avons souhaité construire un terrain d'étude empirique qui mêle une description précise du dispositif avec une analyse des discours des différents acteurs du projet. Ces données sont en outre complétées par les résultats d'une enquête menée sur le terrain ${ }^{1}$. Il s'agit essentiellement de dépasser ces discours parfois stéréotypés qui définissent

1 Ces résultats proviennent d'entretiens semi-directifs menés auprès des commanditaires et producteurs du dispositif ainsi que du recueil sur le terrain des retours et appréciations des usagers après expérimentation de l'outil. Ces données ont été recueillies dans le cadre d'une évaluation des usages réalisée à la demande conjointe du studio de design 2Roqs et de la 
trop souvent le recours aux technologies numériques comme une forme de rupture avec les pratiques traditionnelles. Dépassant les discours technicistes des concepteurs qui parient sur l'efficacité de l'outil par le seul design de l'interface ( « intuitive », « magique »), nous forgeons l'hypothèse que l'usage de la réalité augmentée pour valoriser le patrimoine urbain doit être replacé dans la complexité de la pratique touristique traditionnelle. Celle-ci s'appuie notamment sur un rapport particulier à l'espace physique de la ville (scruter, observer, écouter, etc.) qui doit être intégré dans la conception du dispositif. Cette étude permet d'autre part d'interroger les notions d'immersion et de réalité augmentée exploitées pour définir cette expérience.

Après avoir rappelé le processus de transformation patrimoniale subi par l'espace urbain et la matérialisation physique de cette mutation au sein de son réseau et de ses édifices, nous tenterons de définir les interactions avec le passé qui sont proposées à l'usager à travers cet outil numérique. C'est au cours de cette seconde partie que nous ferons plus largement appel aux données recueillies sur le terrain. Dans une ville aujourd'hui muséifiée, nous nous interrogerons in fine sur cette nouvelle écriture de l'expérience de visite qui valorise un rapport de plus en plus intériorisé et informationnel au patrimoine urbain.

\section{Le patrimoine urbain, entre fixité du monument et mobilité de la visite}

L'attractivité touristique du patrimoine, toujours plus menacé par l'urbanisme croissant et parallèlement toujours plus valorisé, lui confère une place à part au sein des politiques culturelles des institutions publiques qui font désormais la part belle aux raisonnements économiques. Au cœur des grandes métropoles, le patrimoine monumental et sa valorisation, culturelle tout autant qu'économique donc, impose dès lors la visite comme activité particulièrement visible au sein des centres villes (Bossé, 2010).

Cette pratique est par ailleurs indissociable du processus de patrimonialisation, mouvement qui suppose en effet que les populations reconnaissent comme patrimoine tout monument ayant subi une première consécration scientifique et juridique (Davallon, 2006). Face à l'explosion des manifestations visant à animer ces monuments, des appareils institutionnels s'efforcent de clarifier ces actions en coordonnant par exemple des réseaux professionnels garantissant l'intérêt architectural de tel ou tel ensemble urbain.

\subsection{Du monument à l'ensemble urbain labellisé}

Depuis la reconnaissance internationale de la qualité artistique et historique de son architecture urbaine, la ville de Bordeaux a ainsi vu le nombre de ces visiteurs se multiplier à l'envie ; et cette attractivité est chaque année grandissante. Avec le développement croissant du tourisme culturel, c'est-à-dire d'une «pratique culturelle qui nécessite un déplacement ou que le déplacement va favoriser » (Origet du Cluzeau, 2000), l'espace de la cité est traversé de nouvelles pratiques de découverte qui redéfinissent l'expérience urbaine du Port de la Lune, nom traditionnellement donné au centre-ville de Bordeaux du fait du large méandre en forme de croissant de lune que décrit la Garonne lorsque celle-ci traverse la cité. Formule illustrée sur le blason médiéval de la ville, c'est d'ailleurs en ces termes, « Bordeaux, Port de la Lune », que

Mairie de Bordeaux. Cette dernière s'appuie sur le recueil d'une série de questionnaires remplis auprès d'usagers durant deux périodes, du 20 au 31 juillet et lors des Journées Européennes du Patrimoine, soit du 19 au 20 septembre. Au total 53 personnes ont pu être interrogées. Une observation distanciée de visiteurs en situation d'usage a également été conduite durant le mois de septembre 2015 grâce à la promotion des Master Régie des Euvres et Médiation de l'Architecture et du Patrimoine de l’Université Bordeaux Montaigne. 
l'Organisation des nations unies pour l'éducation, la science et la culture (Unesco) a inscrit le 28 juin 2007 ce vaste périmètre de 1810 hectares sur la liste du patrimoine mondial.

Inscrit au titre d'ensemble urbain exceptionnel, c'est cependant avant tout la ville du XVIII ${ }^{\mathrm{e}}$ siècle qui fait l'objet de cette reconnaissance. Si l'ancienneté du port de Bordeaux, et son influence tant à l'échelle nationale qu'internationale, remonte en effet au moins à l'époque antique et prend un essor considérable durant le Moyen Âge, l'époque des Lumières remanie en profondeur la topographie et l'organisation de la cité portuaire. Cette restructuration urbaine se concrétise par l'élévation à partir de la seconde moitié du XVIII ${ }^{\mathrm{e}}$ siècle de nombreux monuments de styles classique et néo-classique. Fonctionnelle, à l'image des pavillons de la place Royale, aujourd'hui place de la Bourse, qui accueillent toutes les structures administratives dévolues au commerce, cette architecture est avant tout une manifestation symbolique et artistique de l'influence considérable de Bordeaux sur le commerce international. Cette richesse doit se voir dans ces monuments qui, reprenant en cela l'étymologie même du mot, rappellent à la mémoire des voyageurs et visiteurs du siècle des Lumières que le Port de la Lune est alors l'une des principales places économiques d'Europe.

Désormais emblème de la ville, cette place de la Bourse devient, au sens médiologique (Debray, 2000), le moyen de transmission monumental tout autant que la trace intemporelle de cette réussite éblouissante d'une cité provinciale. Devenue mémoire monumentale, celle-ci va conséquemment générer une sorte de protection inconsciente qui influe sur l'évolution urbaine. Très peu bouleversée par le XIX ${ }^{\mathrm{e}}$ siècle, c'est en effet au terme d'un siècle qui voit s'accomplir l'éloignement progressif des activités portuaire du centre-ville, que cette place accède à la consécration patrimoniale. Située en plein cœur des 150 hectares du secteur sauvegardé adopté en 1988, la place de la Bourse représente également le lieu central et surtout identifiable pour le périmètre reconnu par l'Unesco. Dépossédés de leurs usages premiers ${ }^{2}$, les monuments qui ceinturent ce paysage urbain et délimitent la place ne constituent plus dès lors des éléments isolés mais témoignent au contraire d'un ensemble spatial, mémoriel et temporel. Devenus objets de savoir pour les professionnels et experts du patrimoine tout autant que de curiosité pour les habitants et touristes (Merzeau, 1999), ils attirent tout naturellement le visiteur. Une simple recherche «Bordeaux» dans Google images illustre à quel point cette façade monumentale est devenue l'emblème iconique, tout autant que touristique, de la cité portuaire. Et c'est tout logiquement dans l'un de ces édifices que la ville de Bordeaux a installé son Ciap, fort habilement nommé «Bordeaux, patrimoine mondial».

\subsection{Du centre d'interprétation à l'interprétation dilatée}

Développée par Freeman Tilden à la fin des années 1950 à partir du modèle des parcs nationaux américains, la notion d'interprétation se manifeste en France au sein des centres d'interprétation qui fleurissent depuis une quinzaine d'années environ et sont, à la différence de leurs lointains cousins d'outre-Atlantique, plus généralement dédiés au patrimoine monumental et aux espaces mémoriels ou transformés par l'Homme. Pour les professionnels du patrimoine français, un centre d'interprétation peut ainsi être défini comme :

« un espace (généralement) sans collection / à visée de diffusion / d'un patrimoine

/ quelle que soit la nature de ce dernier / maintenu en place (inscrit ou aménagé à

2 Précisons toutefois que l'un des pavillons de la place de la Bourse abrite encore aujourd'hui la Chambre de Commerce et d'Industrie de Bordeaux. 
même un lieu naturel) au sein du site qu'il met en valeur / avec des aménagements très minimes / destiné à accueillir un large public » (Chaumier et Jacobi, $2008: 5$ ).

Parce qu'il ne peut évidemment pas être contenu dans un lieu clos, l'ensemble urbain classé par l'Unesco fait donc l'objet depuis le printemps 2014 d'une exposition permanente installée au rez-de-chaussée d'un des pavillons néo-classiques de la place de la Bourse. Rappelant par son nom le précieux label international, ce lieu dépasse toutefois l'image d'Épinal de la cité portuaire du XVIII ${ }^{\mathrm{e}}$ siècle peuplée de négociants pour replacer dans le temps long l'histoire et la topographie du Port de la Lune. Révélant l'insaisissable, le parcours muséographique délivre aux visiteurs les clés de lecture nécessaires à une bonne compréhension de ce vaste ensemble urbain emblématique d'une époque révolue. L'objectif est en outre d'inciter le touriste comme le Bordelais à arpenter la ville de façon autonome et intelligente, avec force documents, prospectus, guides papier ou dépliants. Tout au long de son parcours de découverte, celui-ci est de fait encouragé à utiliser différents dispositifs d'interprétation qui combinent des outils traditionnels, tels que des plans et des maquettes, avec des multimédias interactifs et des audiovisuels aux scénarios recourant plus à la personnalisation du passé qu'à la seule cognition historique. Transformant le monument en document, le centre d'interprétation confronte ainsi le visiteur avec les concepts et hypothèses scientifiques qui façonnent les connaissances actuelles sur l'histoire de la ville tout autant qu'avec un certain vécu du Port de la Lune. En «fictionnalisant» par exemple des récits de personnages historiques évoquant leur vision de Bordeaux, il s'agit pour les concepteurs de renvoyer le visiteur à sa propre vision de la cité portuaire, vision qu'il possède déjà où qu'il se créera en sillonnant son centre historique et ses ruelles pittoresques. La scénographie générale de l'exposition participe donc de ce mouvement qui invite le touriste, selon les termes du responsable du Ciap, à un « voyage à travers le temps et la géographie urbaine de la ville ».

Face à la fugacité des déplacements en situation de visite, l'objectif de cet établissement est en définitive d'ancrer au cœur de l'espace urbain patrimonial «un lieu pour communiquer et “conscientiser" le public » (Chaumier et Jacobi, 2008 : 9) en orientant son regard, l'autorisant parallèlement à dépasser la seule consommation visuelle et corporelle des objets monumentaux pour atteindre une perception du tissu urbain plus compétente. Or, face à des touristes semblet-il toujours plus équipés et toujours plus en quête de liberté, cette communication se dilate et s'étire aujourd'hui à l'extérieur de ce lieu clos. Avec «Bordeaux, passé augmenté », l'exposition se manifeste désormais hors les murs du centre d'interprétation pour prendre vie au sein de l'espace public de la cité à travers l'affichage de documents sur les vitres du monument combiné à l'exploitation d'un dispositif numérique de visualisation. Si les pouvoirs publics et les producteurs de ces systèmes insistent généralement sur la dimension libertaire qu'ils confèrent à la pratique touristique, il convient cependant de dépasser ces discours sur les potentialités de l'outil pour comprendre plus finement l'expérience effective proposée par ce dispositif numérique.

\section{La représentation de la ville comme interface, entre déconnection urbaine et augmentation informationnelle}

Le dispositif développé par le studio de design interactif 2Roqs s'appuie donc sur l'extension de l'exposition permanente du Ciap à l'extérieur de l'édifice. Cette dilatation du centre d'interprétation s'est manifestée par l'affichage sur la façade vitrée du site d'une série documentaire composée de neuf photographies anciennes, sélectionnées, numérisées et imprimées en très grand format. Le commissariat d'exposition a été confié à Frédéric Laux, 
directeur des Archives municipales de Bordeaux. Celui-ci a notamment proposé une thématique capable de fédérer les intentions du projet (usages de photographies anciennes et visite urbaine) ; «Bordeaux, passé augmenté » est ainsi sous-titré «Permanence et mutations des espaces publics ».

\subsection{L'emboîtement des interactions documentaires ou l'exploration déconnectée de la ville}

De ce rapprochement avec les Archives municipales est né un dispositif hybride de valorisation patrimoniale combinant une exposition analogique et une application numérique disponible sur terminaux mobiles (smartphones et tablettes tactiles) ${ }^{3}$. Mais cette hybridation n'est pas sans complexifier l'appréhension du dispositif. Alors que l'exploitation de la réalité augmentée en contexte patrimonial a peu à peu imposé le mythe d'une superposition immersive et transparente de deux visions, une vue actuelle filmée par le terminal mobile et une vue du même lieu restitué (Bordeaux et Renaud, 2014), ici le visiteur ne pointe pas l'espace réel mais une photographie ancienne affichée sur la façade monumentale du Ciap. En d'autres termes, le visiteur vise non pas l'espace urbain mais une représentation de cet espace urbain qui active l'ouverture d'une scène construite à partir du même document d'archive. Par cet emboîtement documentaire, l'usager est de fait complètement déconnecté de son environnement réel, très loin des pratiques que l'on peut constater dans la visite urbaine traditionnelle avec guide conférencier par exemple où l'observation et l'écoute forment le schéma d'acquisition de l'espace. Cette déconnexion se retrouve dans les retours formulés par les visiteurs. Ainsi près de $50 \%$ des personnes ayant répondu au questionnaire délivré par les personnels d'accueil après expérimentation de l'outil n'ont semble-t-il pas apprécié l'usage en extérieur de l'application, estimant que cette consultation pouvait tout aussi bien être réalisée à l'intérieur d'un espace clos assurant plus de confort à la lecture. De même, pour 22\% des personnes interrogées l'environnement urbain a été vécu comme un élément perturbant l'expérience d'immersion pourtant promise par les discours des producteurs et commanditaires.

Car c'est en effet l'objectif principal visé par le projet ; il s'agit pour le concepteur du dispositif de «plonger le visiteur dans la profondeur des photographies anciennes » et de proposer littéralement «un voyage au début $\mathrm{du} \mathrm{XX}^{\mathrm{e}}$ siècle». Pour répondre à cette attente, les photographies, couvrant en réalité une période s'étalant de 1865 à 1910, ont été sélectionnées, non seulement pour leurs sujets, mais aussi et surtout pour leur qualité artistique et documentaire. La présence de personnages au sein des scènes immortalisées a ainsi souvent constitué un critère déterminant pour le choix d'une photographie, assurant de fait une certaine humanisation des représentations élaborées, à l'image de ce que le visiteur peut retrouver durant sa découverte du centre d'interprétation. La sélection effectuée démontre donc une continuité entre les représentations de la ville de Bordeaux véhiculées au sein du Ciap et celles proposées par le dispositif : une ville riche, monumentale, en chantier permanent grâce à un port animé qui fait sa fortune. Dans cet esprit, et pour compléter la modification du document d'archive en espace fictionnel, une série de sons évoquant cette fin du $\mathrm{XIX}^{\mathrm{e}}$ et début du $\mathrm{XX}^{\mathrm{e}}$ siècle a été rajoutée. Bruits des sabots de chevaux sur les pavés, tintements des tramways, foules discutant, autant d'éléments sonores qui viennent animer la scène par des effets plus décoratifs que réellement didactiques.

3 Un site internet a également été créé mais il ne constitue pas à l'origine le cœur du projet qui a été pensé pour être vécu au sein de l'espace urbain : http://goo.gl/Z6V9xF (décembre 2015) 
Grâce à l'ensemble de ce traitement, chaque scène photographique exposée en deux dimensions sur la façade du Ciap est également explorable en trois dimensions par l'usager au sein de l'interface numérique. Immobile au sein de l'espace urbain contemporain, dont il est corporellement et visuellement déconnecté, le visiteur déambule à l'intérieur d'un paysage passé et à jamais perdu. Ce système de navigation interactive façonne une mise en présence du visiteur au sein de l'espace historique qui s'appuie finalement sur les quatre composantes nécessaires à l'expérience spatiale de la visite urbaine telles que définies par Anne Bossé $(2010: 369)$ :

«le franchissement, condition de possibilité de l'expérience [...]; l'accessibilité visuelle, condition de jouissance de l'expérience [...] ; la tension du subir et de l'agir, condition d'expressivité de l'expérience [...] ; la dynamique de l'apprentissage, condition de performation de l'expérience comme expérimentation [...]. »

Franchissant la surface plane de la photographie, le voyageur accède à un espace à l'intérieur duquel il déambule à sa guise en même temps qu'il appréhende les gestes nécessaires à la navigation dans cet univers et actionne sur certains points l'ouverture de contenus textuels à valeur informationnelle. En tant qu'expérience de visite, celle-ci surinvestit donc la vue, l'ouïe n'étant finalement sollicitée que dans une perspective esthétisante (ambiance). Si ce constat permet de rapprocher encore une fois la découverte de cet espace simulé avec la pratique de la visite urbaine (Rautenberg, 2008 : 16), pour un grand nombre d'usagers, des commentaires audio auraient été préférables à la lecture des courts textes délivrant les informations historiques et activables manuellement.

Car si finalement, l'association de la perspective avec l'ambiance sonore fonde la construction d'un environnement visuel au sein duquel l'usager est invité à voyager, celui-ci l'isole dans le même temps de l'espace urbain arpenté. Il convient dès lors de s'interroger sur les ressorts de la visite urbaine qui se voient réellement augmentés par ce dispositif.

\subsection{Entre promesse d'immersion et augmentation de la visite urbaine, un régime scopique en construction}

Techniquement, le recours à la réalité augmentée permet le lancement automatique de l'application et de la scène concernée lorsque le visiteur vise avec son terminal mobile l'une des reproductions photographiques exposées sur la façade du Ciap. Agissant comme un ressort magique, cette transparence logicielle est largement valorisée par le discours promotionnel du projet qui compare le terminal mobile à de véritables «fenêtres ouvertes sur la passé » ${ }^{4}$. Il s'agit ici de vanter un dispositif léger, intuitif et promettant aux visiteurs une expérience inédite d'immersion dans un temps révolu.

Décors restituant la perspective, navigation au sein d'un univers tridimensionnel, vision subjective ; autant d'éléments d'écriture qui assurent à première vue «l'intégration sensorielle de l'usager dans un univers virtuel généré informatiquement $»^{5}$. Et effectivement les quelques propos recueillis auprès des visiteurs témoignent du succès de cette notion d'immersion qui semble aujourd'hui totalement intégrée à l'imaginaire technologique des publics. La grande

\footnotetext{
4 L'écritures des textes commentant cette série documentaire a été menée par l'équipe des Archives Municipales et supervisée conjointement par le Ciap et le studio 2Roqs.

5 Définition de l'immersion virtuelle donnée par la plateforme 100 notions pour le crossmédia et le transmédia, en ligne (consultée en décembre 2015) : http://goo.gl/dycgiR
} 
majorité des personnes interrogées a ainsi estimé que cette expérience les a réellement plongées dans le passé, certains comparant d'eux-mêmes celle-ci à un voyage dans le temps. Pour autant, cette observation relève plus selon nous de la perméabilité des publics aux récits médiatiques qu'à une véritable immersion corporelle réellement vécue par l'ensemble des visiteurs ; les critiques formulées à l'encontre de l'intégration du dispositif au sein du tissu urbain et rappelées ci-dessus obligent en effet à nuancer ces propos enthousiastes. Le sentiment de projection ressenti nous paraît donc devoir être rapproché de la mobilisation attentionnelle (Amato, 2008) que suppose l'utilisation de l'outil et qui transforme l'usager en un sujet regardant un écran et non plus son environnement urbain.

Aucune scène ne représentant en effet la Place de la Bourse depuis le point de vue où se situe physiquement le visiteur, présent et passé s'interpénètrent finalement dans un continuum, non pas topographique ou corporel, mais thématique et expérientiel. Celui-ci se fonde plus particulièrement sur l'exploration de documents numériques et historiques au sein d'un territoire touristique. L'augmentation est avant tout informationnelle, puisqu'elle « consiste en une certaine façon d'articuler de l'expérience (visiter, se repérer, regarder, écouter) et des informations supplémentaires sous la forme de documents (visuels, sonores) » (Bordeaux et Renaud, 2014 : 166). Plus que l'expérience urbaine du visiteur qui, comme nous l'avons déjà souligné, est déconnecté de l'espace où il se trouve physiquement, ce sont donc surtout les contenus informationnel et communicationnel des représentations sélectionnées qui se voit augmenté par l'ajout d'informations historiques et d'artifices esthétiques censés immerger celui-ci au XIX ${ }^{\mathrm{e}}$ siècle. Il s'agit finalement pour les concepteurs d'augmenter les compétences scopiques des publics en leur permettant d'acquérir de nouvelles connaissances sur la ville, des connaissances autres que la vision traditionnellement associée à celle-ci depuis son inscription Unesco, celle d'un modèle de ville néoclassique hérité du XVIII ${ }^{\mathrm{e}}$ siècle, des connaissances qui lui permettent enfin de suivre les permanences et les modifications de l'espace urbain bordelais aux $\mathrm{XIX}^{\mathrm{e}}$ et $\mathrm{XX}^{\mathrm{e}}$ siècles.

De fait, si les régimes scopiques impliquent « une disposition particulière du corps dans l'espace, une gestion du mouvement, un déploiement des corps individuels, tous corrélats qui codifient et normalisent » notre façon de regarder (Jay et Albaret, 1993), alors à n'en pas douter «Bordeaux passé augmenté » façonne un système de consommation visuelle en cours de construction et encore loin d'être codifié. Cette réalisation, en se concentrant uniquement sur les interactions de l'usager avec l'outil numérique produit, ne semble à cet égard pas avoir pris en compte l'ensemble de l'espace urbain et les conditions d'interaction traditionnelle entre le visiteur, la ville d'hier et celle d'aujourd'hui, interactions qui s'appuient sur une forme d'engagement corporel et visuel puisqu'il s'agit avant tout de se repérer, d'observer et, enfin, d'accéder à des informations via un tiers médiateur (guide ou dispositif documentaire).

\section{Conclusion :}

La visite patrimoniale « outillée » active visiblement deux formes de perception de l'espacetemps qui mêlent localisation au sein du territoire pour lequel le voyageur s'est déplacé avec exploration d'une documentation numérique illustrant une partie de territoire. Cette double sollicitation provoque une errance complexe où, immobile physiquement le temps de sa consultation documentaire, le touriste arpente visuellement un univers disparu. Cette mobilitéimmobile interroge en définitive ce qui fait sens pour des usagers qui, pour au moins $30 \%$ d'entre eux, ont manifesté le désir d'être accompagnés dans leur découverte par un médiateur formé et expérimenté, capable de personnaliser cette découverte patrimoniale et donc de 
faciliter la transmission d'une certaine mémoire de la cité. Or, le système de valorisation patrimoniale présentée ici confronte deux formes de mémoires, l'une monumentale et témoignant du XVIII ${ }^{\mathrm{e}}$ siècle, l'autre documentaire et provenant de la fin du $\mathrm{XIX}^{\mathrm{e}}$ siècle, dont il est difficile de cerner l'articulation spatiale tout autant que temporelle du fait de cette errance complexe et protéiforme.

Parce qu'on ne visite enfin jamais seul, «le visiteur vient aussi pour voir avec les yeux des autres » (Bossé, 2010 : 242) - ceux du guide, ceux du groupe avec lequel nous visitons, etc. ; et c'est ainsi que s'élabore finalement une mémoire spatialisée et collective commune des espaces patrimoniaux. Or, ces nouvelles formes de parcours technicisées individualisent la réception de l'information qui ne favorise guère a priori la relation à l'autre (Pagès, 2010), comme l'attestent les postures des usagers immortalisées lors des observations menées.

On assiste ainsi à un processus d'intériorisation d'une expérience de visite hybride qui semble installer une certaine distance avec le territoire urbain tout autant qu'avec les pratiques patrimoniales habituelles qui sont au cœur du tourisme culturel où, traditionnellement, la figure centrale du guide demeure à l'exacte interface entre visiteurs et patrimoine (Sagnes, 2010). Or, pour que ces dispositifs n'augmentent pas seulement l'information mais deviennent pour le touriste un véritable «outil de transition, de transposition et de transaction entre l'espace personnel du visiteur et le lieu de passage » (Gentès, 2008, 290), il convient, nous semble-t-il, que le design ne se concentre plus seulement sur l'interface reliant l'outil et son usager mais aussi et surtout sur l'écosystème global qui prévaut dans la pratique de la visite patrimoniale. Territoire et médiateur humain, au sens d'intermédiaire justement, nous paraissent notamment devoir être remis au centre de la réflexion lors de la conception de ces dispositifs de visite en réalité augmentée.

\section{Bibliographie :}

Amato, É. A. (2008) : Le jeu vidéo comme dispositif d'instanciation, du phénomène ludique aux avatars en réseau, Thèse de Doctorat en Sciences de l'Information et de la Communication, Université de Paris 8.

Bideran, J. (de) (2014), « Visite numérique et parcours augmenté, ou les interactions complexes des touristes avec le patrimoine », Échappées (2), p. 38-48.

Bordeaux, M.-C. et Renaud L. (2014), «La reconfiguration symbolique des territoires touristiques par la réalité augmentée : nouvelles écritures des visites patrimoniales », in L. Fournier, S. Laurent Sébastien, C. Chastagner et C. Bernié-Boissard (dir.), Les cultures du déplacement, Aix-en-Provence, PUAM.

Bordeaux, M.-C. et Renaud L. (2012), «Patrimoine augmenté et mobilité, vers un renouvellement de l'expérience culturelle du territoire », Interfaces numériques (2), p.273-285.

Bossé, A. (2010) : L'expérience spatiale de la visite, engagement dans l'action, épreuve collective et transformations urbaines, Thèse de Doctorat en Géographie, Université François Rabelais, en ligne (consulté en décembre 2015) : http://goo.gl/SMc9xa 
Chaumier, S. et Jacobi, D. (2008) : «Nouveaux regards sur l'interprétation et les centres d'interprétation », La Lettre de l'OCIM (119), en ligne (consulté en décembre 2015): http://goo.gl/N9WWSa

Debray, R. (2000) : Introduction à la médiologie, Paris : Presses universitaires de France.

Davallon, J. (2006), Le don du patrimoine: une approche communicationnelle de la patrimonialisation, Paris, Hermès science - Lavoisier.

Frechin, J.-L. (2008), «Interfaces : un rôle pour le design », in B. Stiegler (dir.), Le design de nos existences à l'époque de l'innovation ascendante, Paris, Fayard, Mille et une Nuits, p. 253265.

Gentès, A. (2008) : « Musée et technologies mobiles : une nouvelle cour du visiteur », in B. Stiegler (dir.), Le design de nos existences à l'époque de l'innovation ascendante, Paris, Fayard, Mille et une Nuits, p. 287-291.

Lesaffre, G., Watremez, A. et Flon, É. (2014), «Les applications mobiles de musées et de sites patrimoniaux en France : quelles propositions de médiation ? », La Lettre de l'OCIM (154), en ligne (consulté en septembre 2014) : http://goo.gl/WNx513

Martin, J. et Albaret M. (1993), «Les régimes scopiques de la modernité », Réseaux, 11 (61), p. 99-112.

Merzeau, L. (1999) : «Du monument au document», Cahiers de médiologie (7), en ligne (consulté en décembre 2015) : http://goo.gl/vPnmX9

Origet du Cluzeau, C. (2000) : Le tourisme culturel, Paris, PUF, Coll. Que sais-je.

Pagès, D. (2010) : «Les territoires numériques : au-delà de l'information localisée, l'hospitalité au fil des écrans?», Quaderni (72), en ligne (consulté en décembre 2015): $\underline{\text { http://goo.gl/hWvPmr }}$

Rautenberg, M. (2008) : «Introduction », L'imaginaire de la ville, le regard et le pas du citadin (Sous la direction de Michel Rautenberg), Culture \& Musées (12), p. 13-29.

Sagnes, S. (2010) : « Suivez le guide... : de 1'Autre à soi, ou comment devenir monument », Ethnologies (32), en ligne (consulté en décembre 2015) : http://goo.gl/c8GVtw

Vial, S. (2014) : «Le design, un acte de communication ? », Hermès (70), p. 174-180. 